\title{
Mesoscopic Fluctuations in Small Metal Particles Studied by Nuclear Magnetic Resonance
}

\author{
F. C. Fritschij, H. B. Brom, and L. J. de Jongh \\ Kamerlingh Onnes Laboratory, Leiden University, P.O. Box 9504, 2300 RA Leiden, The Netherlands \\ G. Schmid \\ Institut für Anorganische Chemie, Universität GH Essen, Universitätsstrasse 5-7, D-45117 Essen, Germany
}

(Received 2 October 1998)

\begin{abstract}
In assemblies of metal nanoparticles small random perturbations are predicted to lead to (a) a statistical energy level distribution around the Fermi level within the assembly and (b) local electron density fluctuations within each particle. ${ }^{195} \mathrm{Pt}$ relaxation experiments on monodisperse 1.2-nm-diam $\mathrm{Pt}$ cores in molecular metal clusters $\mathrm{Pt}_{309} \mathrm{Phen}_{36}^{*} \mathrm{O}_{30}$ agree with these predictions, whereas for the NMR line shape the presence of (b) is obscured by additional contributions from surface effects. [S0031-9007(99)08668-8]
\end{abstract}

PACS numbers: 73.23. $-\mathrm{b}, 71.24 .+\mathrm{q}, 71.30 .+\mathrm{h}, 76.60 .-\mathrm{k}$

In a small metal particle (SMP) of $N$ atoms the energy splittings $\Delta$ around the Fermi energy $E_{F}$ are of order $\Delta \sim E_{F} / N$. At thermal energies much larger than the interlevel spacings the system will behave like a bulk metal, but with decreasing temperature the gaps between levels can no longer be neglected and deviations from bulk behavior become predominant (quantum-size effect). Clear experimental observation of such a crossover from bulk to quantum size regime has been the goal of many experiments on assemblies of SMP's from the very beginning a few decades ago [1]. Such assemblies (required for sufficient experimental sensitivity) were always characterized by substantial size distributions of the particles, blurring the results. But even in an assembly of particles of uniform size (monodisperse), the precise energy level structure is expected to differ between particles due to such small perturbations as surface roughness and randomness in the packing, leading to a distribution in $\Delta$ values [2]. For the assembly the energy gap then becomes a pseudogap and to predict the thermodynamic behavior one has to use statistical theories for energy level distributions [3], such as the random matrix theory [4-6]. As realized a few years ago, not only the variation in the energy density of states (DOS), but also multiple scattering leading to local electron density fluctuations can be very important for the thermodynamic properties [7]. To enable a clear separation of the thermodynamic behavior arising from these mesoscopic statistical effects from the effects due to size distribution, the availability of metal particles of uniform size is a conditio sine qua non. Recently evidence for quantum-size effects in the electronic specific heat and susceptibility was obtained in a series of molecular Pd clusters and colloids [8]. Here we present the NMR properties of a related monodisperse Pt cluster compound. The conditions of uniform core size, surface roughness, and random packing together with electron exchange (see below) allow us to obtain a detailed test of the mesoscopic predictions for nanoparticles for the first time. It is demonstrated that the statistical distribution of the energy levels goes hand in hand with strong local fluctuations in the electron density within each metal cluster.

The nuclear relaxation rate $T_{1}^{-1}$ is a convenient probe for the energy DOS, while the NMR line shape is unique in its sensitivity for the local electron density. For example, in bulk metallic samples, which have a large density of delocalized states at $E_{F}$, the product $T_{1} T K^{2}$ is constant (the well known Korringa relation). In normal metals (such as Pt) the Knight shift $K=\Delta B / B_{0}$ - the relative shift of the line due to the conduction electrons [9] - is $T$ independent. For SMP's, Efetov and Prigodin [10], using a supersymmetry method, and Beenakker [11], using random matrix theory, have shown how the large density fluctuations due to multiple scattering make $K$ dependent on $T$ and change the NMR line profile. For the SMP we selected the metal cluster compound [12,13] $\mathrm{Pt}_{309} \mathrm{Phen}_{36}^{*} \mathrm{O}_{30}$ (abbreviated as $\mathrm{Pt}_{309}$ ) having a core of $309 \mathrm{Pt}$ atoms with a diameter $d$ of $2.1 \mathrm{~nm}$, surrounded by ligand molecules; Phen* [12] indicates a 1,10 phenanthroline derivative. The mean energy splitting $\delta=\langle\Delta\rangle$ at $E_{F}$ can be estimated from $\delta \sim E_{F} / N$ or by using the bulk density of states per atom $\left(1.55 \times 10^{-4}\right.$ states $/ \mathrm{K}$ atom $)$; the resulting values are, respectively, 60 and $40 \mathrm{~K}[1,14]$. The cluster molecules form a randomly packed noncrystalline solid. Because of this randomness, electron exchange between Pt cores proceeds via thermally activated hopping [15].

In systems with $I=1 / 2$ nuclei like ${ }^{195} \mathrm{Pt}$ used here as a probe, complications due to quadrupolar interactions are avoided and the relaxation rate $T_{1}^{-1}$ is twice the transition rate $W$ between the two nuclear Zeeman levels. When the electronic energy levels are discrete and have a Lorentzian broadening with half width at half height $\gamma$, the rate $W$ depends on the occupation, $p(E)$, of initial and final energy states ( $E_{i}$ and $E_{f}$, respectively) and the overlap on these levels (energy conservation) [16]:

$$
W=A \sum_{i, f} p\left(E_{i}\right)\left[1-p\left(E_{f}\right)\right] \frac{\left(\gamma_{i}+\gamma_{f}\right)}{\left(\gamma_{i}+\gamma_{f}\right)^{2}+\left(E_{i}-E_{f}\right)^{2}},
$$


with $A$ a proportionality constant. In metals, where the density of states $D(E)$ is quasicontinuous, $T_{1}^{-1} \propto$ $\int_{0}^{\infty} D(E)^{2} f(E)[1-f(E)] d E \approx D\left(E_{F}\right)^{2} k_{B} T$, where $f(E)$ is the Fermi Dirac distribution function $[9,16]$. Hence $T_{1}^{-1} \propto T$, which will also hold in SMP's as long as $\Delta \ll k_{B} T$. The level broadening due to excitations within the particle such as intracluster vibrations or from intercluster processes such as phonon-assisted electron hopping between particles becomes essential for $\Delta \gg k_{B} T$. In a low- $T$ approximation, we restrict the summation in Eq. (1) to two levels only and the expression for the magnetization recoveries $M(t)$ after saturation is given by

$$
M(t)=\int M_{0}\left\{1-\exp \left[-t / T_{1}(\Delta)\right]\right\} P_{0}(\Delta) d \Delta .
$$

Here $P_{0}(\Delta)$ is the distribution function for the energy splitting $\Delta$ between the two levels [3]. The influence of other nearby levels and the electron exchange between particles can be approximately accounted for by an effective $\Delta$ and $T$ dependence of the $\gamma$ 's. For example, thermally activated excitations between energy levels within or between the particles will produce an activated form of $\gamma \propto \exp \left(-\Delta / k_{B} T\right)$, while for nonactivated tunneling between particles $\gamma \sim \alpha \Delta$, with $\alpha$ a measure of the tunneling rate [10].

In Fig. 1 data for the line shape obtained in frequency $(\nu)$ and field $(B)$ sweeps are shown for representative temperatures. As in other studies on Pt particles [17-19], the width is seen to be very broad and $T$ independent. The MHz width of these line profiles is in sharp contrast to those found in bulk Pt (about $30 \mathrm{kHz}$ in $9.4 \mathrm{~T}$ ) or in simple chemical $\mathrm{Pt}$ compounds (a few $\mathrm{kHz}$ ) and are typical for SMP's. In Fig. 2a we show $T_{1}$ at $85.55 \mathrm{MHz}$

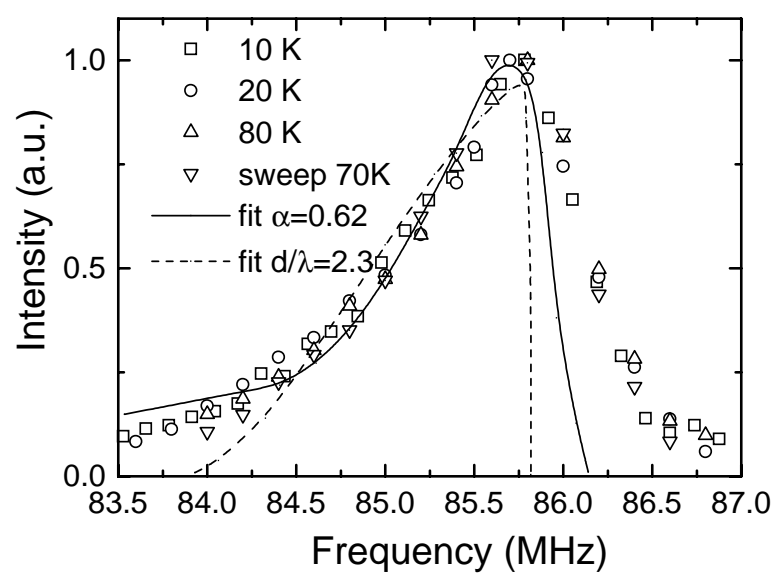

FIG. 1. The line intensity of $\mathrm{Pt}_{309}$ at various $T$ 's measured by frequency sweeps at $B=9.4 \mathrm{~T}$. Drawn and dashed lines are fits discussed in the text. To check the possible influence of circuit retuning needed during these sweeps, we also performed a field sweep at $67.65 \mathrm{MHz}$ and $70 \mathrm{~K}$. Using the gyromagnetic ratio these data are translated to frequency sweeps at $9.4 \mathrm{~T}$. After translation all data coincide. In $9.4 \mathrm{~T}$ the bulk $\mathrm{Pt}$ resonance is at $82.6 \mathrm{MHz}$, while the chemical shifts for $\mathrm{Pt}$ compounds lie around $86 \mathrm{MHz}$. in $9.4 \mathrm{~T}$ as a function of temperature for $T>80 \mathrm{~K}$, i.e., higher than the average level spacing. Because the linewidth is much broader than the bandwidth of the $\pi / 2$ pulse, the frequency at which $T_{1}$ has been measured must be specified. The frequency dependence of $T_{1}$ at $80 \mathrm{~K}$ is shown in Fig. 2b. The data were checked to be independent of the length of the $\pi / 2$ pulse. At all scanned frequencies above $65 \mathrm{~K}$ the relaxation is single exponential [20] and the spin lattice relaxation time is inversely proportional to temperature ( $T_{1} T$ is constant).

In bulk platinum $T_{1}$ has contributions from $s$ and $d$ electrons and an orbital contribution. The calculations of Refs. $[18,19]$ transpose the bulk behavior to Pt SMP's by introducing site-dependent $s$ and $d$ densities of state due to the small size. From the combined measurements of the Knight shift $K$ and $T_{1}$, the $s$ and $d$ density of states can be computed. The single exponentiality of the $M(t)$ recoveries implies that at different frequencies only one combination of $s$ and $d$ density of states $\left(D_{s}\right.$ and $D_{d}$, respectively) is involved. The solid line in Fig. $2 \mathrm{~b}$ is a fit where it is assumed [19] that only the $d$ part of the Knight shift is distributed; the orbital contribution is fixed at the bulk value $\left(K_{\text {orb }}=0.21 \%\right)$. An equally good fit can be obtained by including a healing length $\lambda$ for the $s$ electron density as well [18]. From the decomposition of the total Knight shift at a given frequency and the fit to the relaxation rates, $D_{d}$ and $D_{s}$ are found. $D_{s}\left(E_{F}\right)$ equals 0.28 states $\mathrm{eV}^{-1}$ atom ${ }^{-1}$, close to the bulk value of 0.30 states $\mathrm{eV}^{-1}$ atom ${ }^{-1}$. The bulk value of $D_{d}\left(E_{F}\right)$ (reached far from the surface) is 5 times larger than $D_{s}\left(E_{F}\right)$. In this analysis the bulk Korringa constant $5.59 \times 10^{-6}$ sK is used.

In the mesoscopic calculation of the line shape $L(\nu)$ as a function of $\nu[10,11]$, the Knight shift $K$ has to be weighed by the number of nuclei having that particular shift. The mesoscopic treatment for the unitary ensemble leads to a simple zero-Kelvin expression for $L(\nu)$, which depends only on one parameter $\alpha$, that is proportional to the ratio of level broadening and mean level splitting [10]; between orthogonal and unitary ensemble only
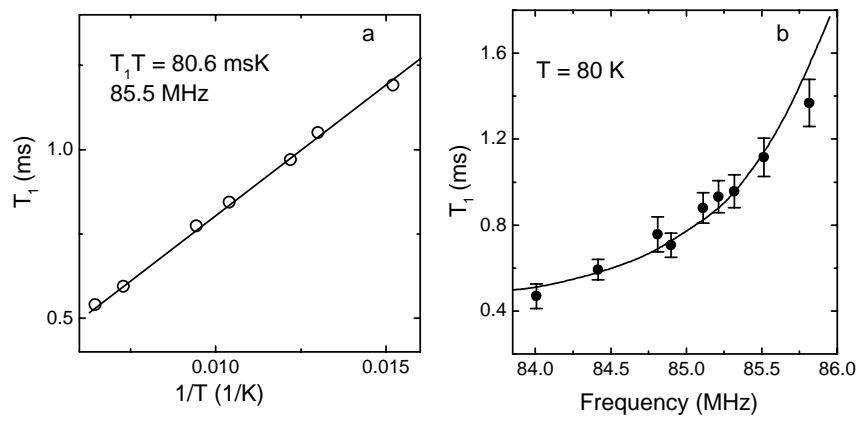

FIG. 2. Relaxation time for $\mathrm{Pt}_{309}$ (a) as a function of $1 / T$ at $85.5 \mathrm{MHz}(65<T<165 \mathrm{~K})$ and (b) as a function of frequency at $80 \mathrm{~K}$. The solid lines in (a) and (b) are fits based on the full Korringa relation [19]; see text. 
small changes are expected [21]. With increasing $T$ the resonance profile is predicted to shift and narrow to the bulk position and shape. Although the measured line shape can be well fitted by the mesoscopic expression [22] — solid line in Fig. 1-the expected temperature dependence is absent experimentally. Why does this prediction fail [16]? In the high temperature limit where the Korringa relation holds, our data show that the line shape is still broad. Calculating $L(\nu)$ in terms of the surface model discussed above for $T_{1}$ and $K$ with the parameters derived from the frequency dependence of $T_{1}$ in this Korringa regime, the line is also well reproduced [14,18,19] (dashed line in Fig. 1). In this surface model the profile is $T$ independent indeed, as it arises from the dominance of the healing lengths of $D_{d}$ and $D_{s}$ [23] and other surface effects. As illustrated by Gascôn and Pastawski [24], these surface effects might indeed overshadow the intrinsic mesoscopic features. The conclusion is that at temperatures higher than $65 \mathrm{~K} T_{1}$ and $K$ of the Pt cores behave as in bulk Pt, with the same Korringa constant but with a reduced and site dependent $d$ density of states. Up to this point, our results are reminiscent of those of other groups [17-19]. In most of these studies the Korringa relation was seen to be obeyed even down to their lowest temperatures of a few degree Kelvin. Only in Pt particles enclosed in cages of zeolites deviations from Korringa fits were seen; restricted breathing mode vibrations due to the confinement in the cages were proposed as an explanation [25].

We now turn to the relaxation behavior observed below $65 \mathrm{~K}$. Figure 3 gives the recoveries of the nuclear magnetization $M(t)$ as a function of the product of time $t$ and temperature $T$ at representative temperatures. By this $\mathrm{T}$ scaling of the time, the recovery curves in the Korringa regime $(T>65 \mathrm{~K}$, the $80 \mathrm{~K}$ data are given as an example) fall on top of each other. Nonexponentiality

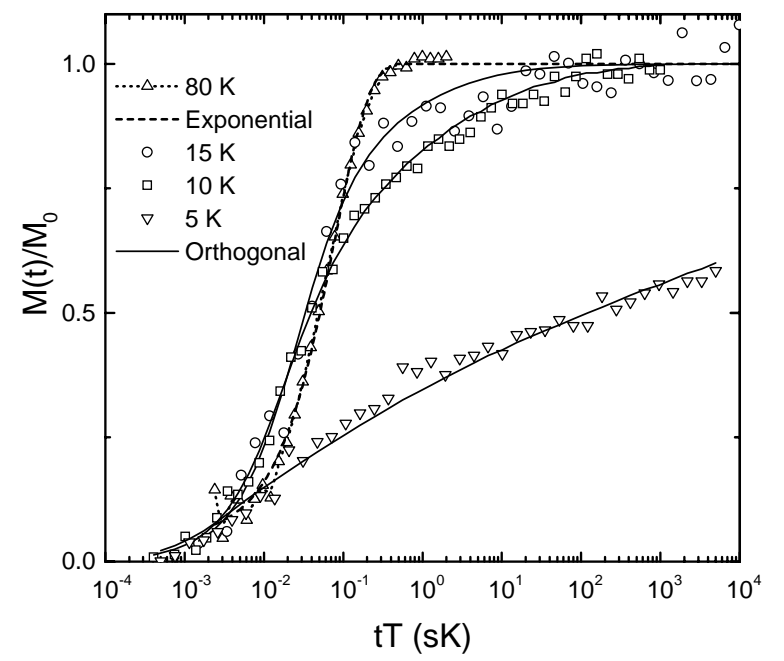

FIG. 3. Recovery of the magnetization as a function of $t T$ for $\mathrm{Pt}_{309}$ and fits with Eq. (2) for the orthogonal level distribution. is observed to start below about $50 \mathrm{~K}$ and becomes strongly pronounced below $10 \mathrm{~K}$. In contrast to Korringa behavior, the scaled curves are now temperature specific. While at $10 \mathrm{~K}$ equilibrium could be reached in an hour, at $5 \mathrm{~K}$ it is estimated that the magnetization needs at least 3 orders of magnitude more time for its return to the thermal equilibrium value $M_{0}(T)$. Accordingly we determined $M_{0}$ at $5 \mathrm{~K}$ from equilibrium data at $10 \mathrm{~K}$, assuming that $M_{0}(T)$ obeys the Curie law. To explain the crossover to nonexponential behavior, we note that the observed crossover temperature is close to the expected average energy level splitting in the Pt core of around $40 \mathrm{~K}$. Qualitatively, this is understood as follows. Measurements are done at a certain frequency, i.e., only nuclei with the same Knight shift, i.e., local electron density are involved. Because of the mesoscopic scattering and surface roughness, electrons in cores with different energy level splittings at $E_{F}$ can still have the same local electron density and hence contribute to the signal. When thermal broadening is no longer sufficient to have a quasicontinuous DOS at $E_{F}$, the differences in energy splitting will become manifest.

To model the nonexponential recoveries of $M(t)$ with their strong temperature dependence we use Eqs. (1) and (2) and assume that the broadening $\gamma$ depends on the level spacing $\Delta$ in the following way:

$$
\gamma(\Delta)=\gamma_{0}(T) \exp \left(-\Delta / T_{e}\right)
$$

with $T_{e}$ an effective broadening temperature. We find good fits (solid lines in Fig. 3) for the orthogonal distribution function $P(x)=\frac{1}{2} \pi x \exp \left(-\frac{1}{4} \pi x^{2}\right)$ with $x=\Delta / \delta$ with $\delta=\langle\Delta\rangle$. As $M(t)$ is insensitive to small changes in $T_{1}(\Delta)$, the population factor $p\left(E_{i}\right)\left[1-p\left(E_{f}\right)\right]$ is set equal to unity. The proportionality constant $A$ appearing in Eq. (1) is found to be constant within a factor of 2 (at $5 \mathrm{~K}, A=5 \times 10^{3} \mathrm{~K} \mathrm{~s}^{-1}$ fits the data slightly better than $\left.3 \times 10^{3} \mathrm{~K} \mathrm{~s}^{-1}\right)$. Values of the prefactor $\gamma_{0}(T)$ are of the order of $10^{3} \mathrm{~K}$ (due to the exponential dependence of $\gamma$ on $\Delta / T_{e}$ only the value at $15 \mathrm{~K}$ of $864 \pm 4 \mathrm{~K}$ is accurate). The effective broadening temperature $T_{e}$ scales with the real temperature $T(7 \pm 4 \mathrm{~K}$ at $15 \mathrm{~K}, 5 \pm 1$ at $10 \mathrm{~K}$, and $1.4 \pm 0.1$ at $5 \mathrm{~K}$ ), which shows that activated intraparticle or interparticle processes dominate the line broadening.

Finally, we address the role of phonons and intercluster electron exchange. Because of the confinement in the metal cluster cores, the spectrum of the intracluster phonon modes also becomes discrete. Such discrete intracluster phonon spectra have been calculated and were found to agree quite well with the experimental specific heats of these cluster compounds [26-28]. The same data sets show that, although below $20 \mathrm{~K}$ the intracore phonons are no longer excited, ligand and intercluster vibrations give rise to a large contribution to the specific heat and are responsible for the thermal equilibrium within the electronic spin system $[8,28]$. As regards intercluster electron exchange, the time scale of this process 
can be estimated from the dielectric measurements of Reedijk et al. [15]. Extrapolating these data to low temperatures suggests that the electronic intercluster as well as intracluster transitions should involve a sufficient number of different cluster cores on the time scale of the NMR relaxation and linewidth experiment to provide a source for the broadening of the energy levels [29].

In summary, in aggregates of $\mathrm{Pt}_{309}$ clusters we find that down to $65 \mathrm{~K} T_{1}$ obeys the same Korringa relation as in bulk Pt. Around $15 \mathrm{~K}$ the recovery curves of $M(t)$ have become strongly nonexponential. This temperature is indeed below the estimated average energy gap at $E_{F}$ for $\mathrm{Pt}_{309}$. The nonexponential recoveries show that within the Pt cluster assembly the energy splittings vary form core to core and that in addition in each Pt core strong local electron density fluctuations are present. The distribution of the splittings is well described by the orthogonal ensemble if thermal broadening of the energy levels is taken into account. In contrast to the relaxation behavior the overall line shape is found to be insensitive to the mesoscopic fluctuations, which can be explained by assuming that it is dominated by surface effects.

We acknowledge C. W. J. Beenakker for useful comments, J. A. Reedijk for the dielectric measurements, and I. Abu-Shiekah for performing NMR on the modified $\mathrm{Pt}_{309}$ cluster compound. The work is part of the research program of the "Stichting voor Fundamenteel Onderzoek der Materie" (FOM) which is partially supported by the "Nederlandse Organisatie voor Wetenschappelijk Onderzoek" (NWO) and has also benefited from support of the European Community under the HCM program.

[1] W. P. Halperin, Rev. Mod. Phys. 58, 533 (1985).

[2] R. Kubo, J. Phys. Soc. Jpn. 17, 975 (1962).

[3] R. Denton, B. Mühlschlegel, and D. J. Scalapino, Phys. Rev. B 7, 3589 (1973).

[4] E.P. Wigner, Proc. Cambridge Philos. Soc. 47, 790 (1951).

[5] F. J. Dyson, J. Math. Phys. (N.Y.) 3, 140 (1962); F. J. Dyson and M. L. Mehta, J. Math. Phys. (N.Y.) 4, 701 (1963).

[6] L.P. Gorkov and G. M. Eliashberg, Sov. Phys. JETP 21, 940 (1965).

[7] B. L. Altshuler, in Nanostructures and Mesoscopic Systems, edited by W.P. Kirk and M. A. Reed (Elsevier, Amsterdam, 1991).

[8] Y. Volokitin, J. Sinzig, L. J. de Jongh, G. Schmid, M. N. Vargaftik, and I. I. Moiseev, Nature (London) 384, 621 (1996).

[9] C.P. Slichter, Principles of Magnetic Resonance
(Springer, Berlin, 1990).

[10] K. B. Efetov and V. N. Prigodin, Mod. Phys. Lett. B 7, 981 (1993); Phys. Rev. Lett. 70, 1315 (1993); K. B. Efetov, Physica (Amsterdam) 83D, 151 (1995).

[11] C. Beenakker, Phys. Rev. B 50, 15170 (1994).

[12] G. Schmid, Struct. Bonding (Berlin) 62, 51 (1985); Clusters and Colloids: From Theory to Applications, edited by G. Schmid (VCH, Weinheim, 1994).

[13] Physics and Chemistry of Metal Cluster Compounds, edited by L.J. de Jongh (Kluwer Academic Publishers, Dordrecht, 1994).

[14] H. B. Brom, D. van der Putten, and L. J. de Jongh, in Ref. [13], p. 227, and references therein.

[15] J. A. Reedijk, H. B. Brom, L. J. de Jongh, and G. Schmid, Phys. Rev. B 57, R15 116 (1998).

[16] J. J. van der Klink, J. Phys. Condens. Matter 7, 2183 (1995).

[17] I. Yu and W. P. Halperin, J. Low Temp. Phys. 45, 189 (1981); Phys. Rev. B 47, 15830 (1993).

[18] C. D. Makowka, C.P. Slichter, and J.H. Sinfelt, Phys. Rev. B 31, 5663 (1985); H. T. Stokes, H. E. Rhodes, P.-K. Wang, C.P. Slichter, and J.H. Sinfelt, Phys. Rev. B 26, 3575 (1982).

[19] J. P. Bucher and J. J. van der Klink, Phys. Rev. B 38, 11038 (1988).

[20] Close to the unshifted resonance position a second Pt line with a different relaxation time appears, which is due to $\mathrm{Pt}$ atoms that are chemically strongly bound to ligand groups [14]; this line is not considered here.

[21] Going from the unitary to the orthogonal ensemble changes the second moment of the line shape at most by a factor of 2 [11].

[22] F. C. Fritschij, Ph.D. thesis, Leiden, 1998.

[23] Slichter et al. [18] consider in addition the effect of spherical boundaries. The corresponding eigenfunctions give strong local density variations for the $s$ electrons on top of the smooth variation in $d$ electron density.

[24] H. M. Pastawski and J. A. Gascôn, Phys. Rev. B 56, 4887 (1997); J. A. Gascôn and H. M. Pastawski, Phys. Rev. B (to be published).

[25] Y. Y. Tong, D. Laub, G. Schulz-Ekloff, A. J. Renouprez, and J. J. van der Klink, Phys. Rev. B 52, 8407 (1995).

[26] H.H.A. Smit, P.R. Nugteren, R.C. Thiel, and L. J. de Jongh, Physica (Amsterdam) 153B, 33 (1988).

[27] J. Baak, H.B. Brom, L. J. de Jongh, and G. Schmid, Z. Phys. D 26, S30 (1993); H. B. Brom, J. Baak, and L. J. de Jongh, in Ref. [13], p. 211.

[28] Y. Volokitin et al., Z. Phys. D 40, 136 (1997); Czech. J. Phys. Suppl. 46, S4, 2373 (1996); Y. Volokitin, thesis, Leiden, 1997.

[29] The importance of broadening due to intercluster processes is confirmed in $\mathrm{Pt}_{309}$ compounds with larger ligands. In these aggregates intermolecular electron exchange proceeds slower and the nonexponential recoveries are seen to start at higher temperatures. 